\title{
OBSERVAC̣ÃO DA VISITA DE MORCEGOS (CHIROPTERA) ÀS FLORES DE PSEUDOBOMBAX GRANDIFLORUM (CAV.) A. ROBYNS ${ }^{1}$
}

\author{
Shirley S. Pereira da Silva ${ }^{2}$ \\ Adriano Lúcio Peracchi ${ }^{2}$
}

\begin{abstract}
OBSFRVATION OF VISIT OF BATS (CHIROPTTRA) TO THE FIOWERS OF PSEUDOBOMbaX GRANDIFLORLM (CAV.) A. ROBYNS. The visit of two species of hats to the flowers of Pseudobombax grandiflorum (Cav.) A. Robyns was observed in the Federal Rural University of Rio de Janeiro during the period of may and july of 1989. The flowers of this vegetal species present attributions in accordance to the Syndrome of Chiropterophily and receive the visit of Glossophaga soricina (Pallas. 1766) and Phyllostomus hastatus (Pallas, 1767). Glossophaga soricina has morfological caracteristes for nectarivory. Phyllostomus hastatus is omnivorous and starts its visits soon after the anthesis. That bats present landing behaviour during the visit,

KEY WORDS. Chiroptera, Pseudobombar grandiflorum, hats, flowers, néctar, chiropterophily
\end{abstract}

Na região tropical vários animais podem desempenhar importante papel na polinização e dispersão de vegetais superiores. Muitas plantas sofreram adaptações morfológicas que facilitaram aos seus visitantes utilizarem os recursos florais oferecidos tais como: néctar, pólen, óleos, etc. Essas alterações demonstram que os vegetais se adaptaram às distintas classes de visitantes/polinizadores (HEITHAUS 1982) e através da morfologia floral pode-se inferir o provável visitante/polinizador de determinado vegetal.

Os morcegos são, entre os mamíferos, os visitantes/polinizadores mais comuns de uma grande variedade de flores, e esta relação denomina-se "Síndrome da Quiropterofilia" e nessa síndrome estão envolvidas espécies de quirópteros do Velho e Novo Mundo. Este processo é extremamente importante na comunidade tropical, pois os morcegos são transportadores de pólen a longas distâncias (BAKER 1973).

Neste trabalho apresentamos as observações da visita de duas espécies de morcegos às flores de Pseudobombax grandiflorum (Cav.) A. Robyns no Campus da Universidade Federal Rural do Rio de Janeiro (UFRRJ).

1) Trabalho elaborado nos laboratórios da Área de Zoologia do Instituto de Biologia da Universidade Federal Rural do Rio de Janeiro. com auxílio do CNPq.

2) Instituto de Biologia, Universidade Federal Rural do Rio de Janeiro. Caixa Postal 745(1)3. 23851-970) Itaguai. Rio de Janeiro. Brasil. Bolsista do CNPq.

Revta bras. Zool. 12 (4): 859 - 865, 1995 


\section{MATERIAL E MÉTODOS}

O Campus está localizado no distrito de Seropédica ( $22^{\circ} 45^{\prime}$ 'S e $43^{\circ} 41^{\prime} \mathrm{W}$ ), município de Itaguaí (Rio de Janeiro), a aproximadamente $60 \mathrm{Km}$ da capital do Estado do Rio de Janeiro e sua área total é de aproximadamente 3024 hectares. A região é baixa e plana, com altitude média de $30 \mathrm{~m}$ e com pequenas elevações espaçadas cujo ponto mais alto atinge $75 \mathrm{~m}$. Apresenta uma grande diversidade florística, onde são encontradas aproximadamente 52 famílias vegetais com várias espécies que servem de alimento para diversos animais (GUIMARĀES 1951).

Coletas semanais foram realizadas de maio a julho de 1989, no período de 17 às 23 horas, com auxílio de redes de espera (mistnets), medindo aproximadamente $12 \mathrm{~m}$ de comprimento, armadas próximo às árvores de Pseudobombax grandiflorum.

Nos morcegos capturados procurou-se observar a presença de material polínico na pelagem que, se presente, era retirado com pincel e acondicionado em frasco de vidro com álcool $70^{\circ} \mathrm{Gl}$. De cada exemplar coletado retirou-se o tracto gastrointestinal completo para observação do seu conteúdo em água destilada sob microscópio estereoscópico. O conteúdo foi coado em tela de cobre de $0,5 \mathrm{~mm}$ de malha para a separação do pólen.

Do material coado e do material proveniente da pelagem foram montadas lâminas palinológicas pelo método direto (gelatina glicerinada). Considerou-se como amostra comprobatória da visita as lâminas que apresentaram mais de 30 grãos de pólen.

Alguns morcegos foram taxidermizados e incorporados à coleção de quirópteros atualmente depositada no Instituto de Biologia da UFRRJ sob os números: ALP - 5297; 5298; 5299. As lâminas palinológicas foram incorporadas à mesma coleção.

As observações noturnas do comportamento dos morcegos ao abordarem às flores foram realizadas ou sob a luz do luar ou com auxílio de lanternas cobertas com papel tipo celofane de cor vermelha. Durante essas observações registrou-se o número de indivíduos visitando às flores e o comportamento de cada animal.

\section{RESULTADOS}

A "Paineira lisa", Pseudobombax grandiflorum é um vegetal de porte arbóreo com aproximadamente sete metros de altura. Apresenta folhas compostas por cinco folíolos que caem totalmente durante o período de floração, deixando os ramos nús e as flores expostas. Essas são grandes, localizadas no extremo dos ramos e possuem de 250 a 280 estames longos, do tamanho das pétalas, brancos, que dá um aspecto de pincel às flores (Fig. 1). O estigma é longo e localiza-se acima do nível das anteras.

A ântese inicia-se às 17:30 horas e durante o processo, as pétalas se enrolam em direção ao pedúnculo floral; paralelamente há eliminação de um odor forte, enjoativo, semelhante a couve fresca.

Durante o período de estudo observou-se que a cada noite o número de 
flores abertas não ultrapassou a cinco e estas são funcionais apenas por uma noite, estando na manhã seguinte os estames murchos. Foi possível observar também a presença de botões, flores e frutos em diversos estágios de desenvolvimento.

Coletaram-se exemplares de Glossophaga soricina (Pallas, 1766) e Phyllostomus hastatus (Pallas, 1767) enquanto visitavam às flores de $P$. grandiflorum.

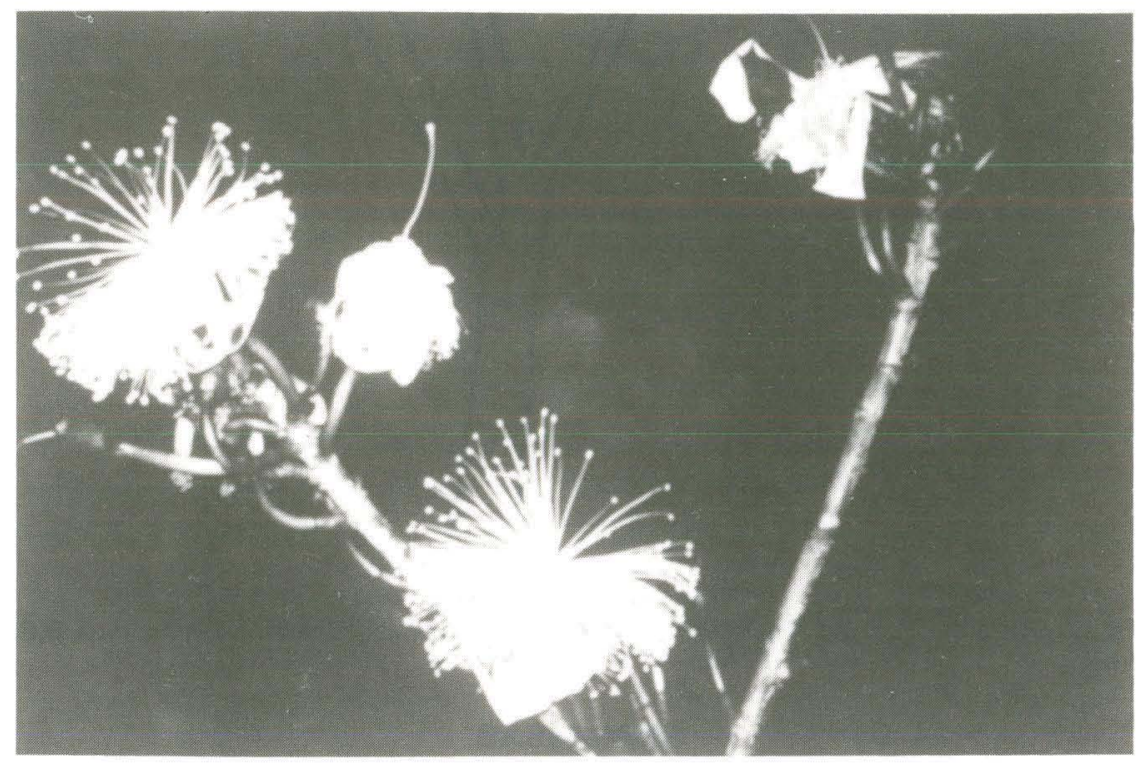

Fig. 1. Flores de Pseudobombax grandiflorum.

A partir das 18 horas foi observada a presença de exemplares de $P$. hastatus. Esses morcegos, de grande porte, aproximavam-se em linha reta do alto da copa, abordavam a flor com um breve pouso com as asas abertas e estendidas para trás, afastando-se e rodeando a copa da árvore. Durante as abordagens os quirópteros agarravam-se ao cálice com as unhas dos pés, introduziam a cabeça entre os estames para recolher o néctar. Após se alimentar, afastavam-se e observou-se a chegada de G. soricina. Essa espécie, de pequeno porte, visitava às flores de $P$. grandiflorum, solitariamente, ou no máximo em dupla. O animal aproximava-se em linha reta, rodeava a copa desnuda, abordava a flor de baixo para cima, pousando sobre ela com as asas recolhidas ou distendidas lateralmente ao corpo (Fig. 2), agarrava-se à flor com as unhas dos pés e polegares e introduzia a cabeça entre os estames para recolher o néctar.

$\mathrm{Na}$ análise das lâminas palinológicas, verificou-se a presença de material polínico de $P$. grandiflorum na pelagem e tracto gastrointestinal dos exemplares coletados (Fig. 3). 


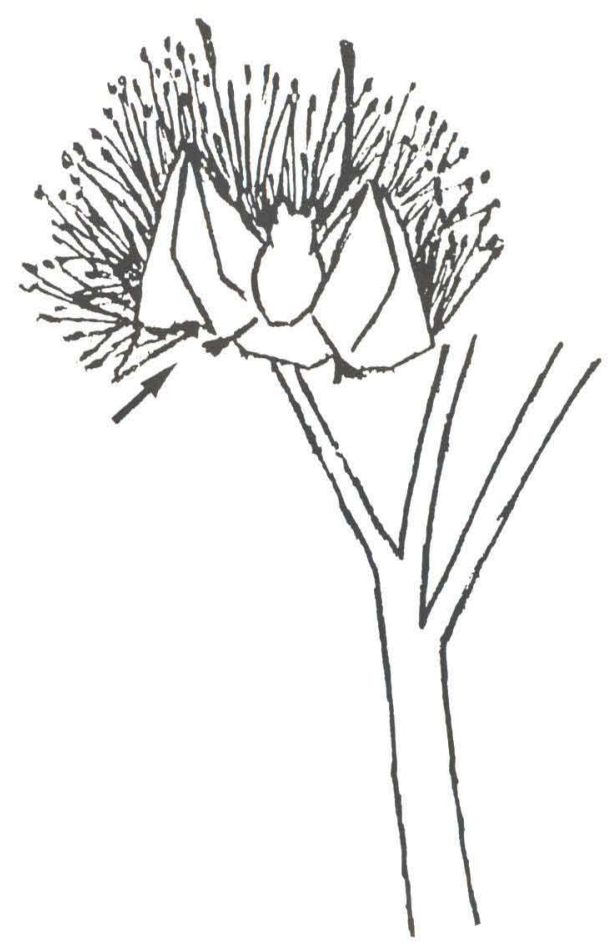

Fig. 2. Visita de Glossophaga soricina, notar as asas recolhidas (seta). Desenho de S.S.P. Silva.

\section{DISCUSSÃO}

Os vegetais que utilizam quirópteros como polinizadores apresentam alguns mecanismos para que esse processo se torne eficiente: ântese noturna, disposição dos elementos florais de forma que o material polínico se deposite na porção anterior do animal (cabeça/tórax), aumento na produção de néctar e pólen, etc (FAEGRI \& PIJL 1971). Isso pode ser observado em Pseudobombax grandiflorum, em cujas flores, os estames, com seu aspecto de pincel, proporcionam um aumento na área de distribuição do material polínico, fazendo com que a cada abordagem dos morcegos, o pólen seja eficientemente disperso na superfície anterior do animal.

RAMIREZ et al. (1984) ao observarem a visita de morcegos às flores de Bauhinia ungulata Benth sugeriram que uma grande produção de néctar promove um alto número de visitas às flores e a deposição do pólen favorece a fertilização cruzada.

Em $P$. grandiflorum a presença de botões, flores e frutos silmutaneamente e o baixo número de flores abertas por noite, pode indicar uma longa florada e assim as flores serem polinizadas pelo sistema de linha de captura, onde o morcego visita flores em uma rota pré-estabelecida a cada noite. 


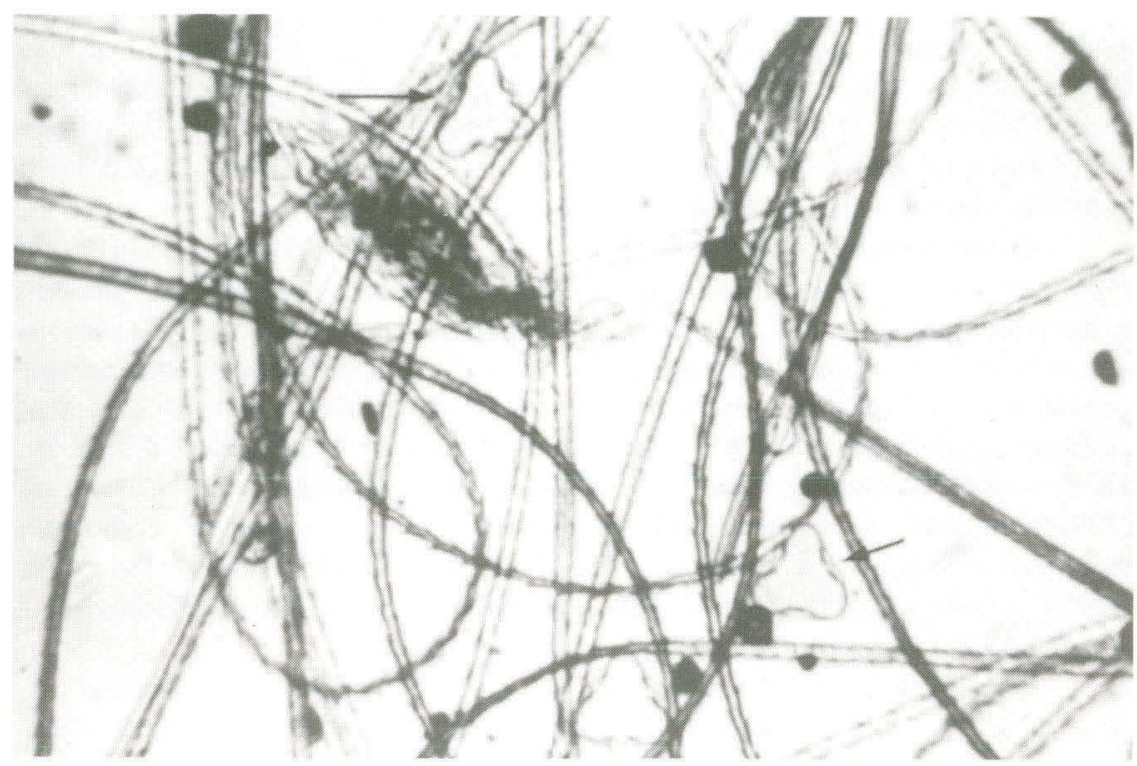

Fig. 3. Material polínico de P. grandiflorum aderido a pelagem de $P$. hastatus (seta).

Das duas espécies de quirópteros observadas, apenas Glossophaga soricina apresenta características morfológicas adaptadas para a nectarivoria tais como: língua longa e extensível, focinho alongado, pelos com projeções que facilitam a aderência do pólen (Howell 1974; Howell \& HodkiNG 1976). Porém, apesar de apresentar uma certa preferência pela nectarivoria, $G$. soricina pode utilizar frutos e insetos (GARDNER 1977).

Phyllostomus hastatus é considerado onívoro (GARDNER 1977) e segundo HOWELL \& BURCH (1974) utiliza insetos e frutos em sua dieta, tendo preferência por Piperaceae e Moraceae. CARVALHO (1961) cita que este morcego utiliza partes florais, incluindo néctar e pólen de Leguminosae e Bombacaceae. Silva (1991) observou este quiróptero utilizando néctar e pólen de Lythraceae (Lafoensia glyptocarpa Koehne).

A alternância alimentar nestas duas espécies pode ser devida ao período de floração dos vegetais, pois a quantidade de recursos alimentares oferecidos torna-se insuficiente para o gasto energético dos animais, devido a alguns vegetais terem um período de floração muito curto, levando-os a uma diversificação.

Assim, P. grandiflorum pode ser considerado como uma grande fonte alimentar para esses morcegos devido ao fato de florescer no inverno, período em que há uma redução na oferta de alimento disponível. Segundo CARVALHO (1961), $G$. soricina alterna sua alimentação buscando qualidade e não quantidade, pois consegue alimento nas flores durante todo ano. 
As flores de $P$. grandiflorum facilitam a visita dos morcegos, não só por estarem localizadas no extremo dos ramos, mas também por oferecerem sustentação suficiente aos animais. O comportamento dos visitantes ao se alimentarem, pousando sobre as flores com o corpo e introduzindo a cabeça entre os estames, possibilita uma dispersão do pólen sobre o corpo, fazendo com que a cada visita aumente a possibilidade de uma polinização.

O comportamento de $P$. hastatus é semelhante ao observado por CARVALHO (1960) em Parkia gigantocarpa Duke, porém as abordagens não são tão violentas. É interessante notar que CARVALHO (1961) não observou a visita de G. soricina às flores abordadas por $P$. hastatus e vice-versa. Sazima \& Sazima - comunicação pessoal a DOBAT (1985) - observaram a visita de Phyllostomus discolor (Wagner) às flores de $P$. grandiflorum. Outros glossofagineos, Anoura geoffrovi (Gray, 1838) e Anoura caudifer (Geoffroy, 1818) foram observados por FISHER et al. (1992) enquanto se alimentavam nas flores de $P$. grandiflorum. Para esses morcegos o comportamento apresentado foi de "adejo" (vôo pairado). Porém, na área de estudo, Glossophaga soricina, que também é um morcego glossofagineo, apresentou um comportamento de pouso ao se alimentar.

O padrão de visitas solitárias ou em dupla parece ser o habitual para $G$. soricina (SAZima \& SAZIMA 1977, 1980; SAZIMA et al. 1982; LemKE 1984), porém CARvalho (1960) e Silva (1991) puderam observar até 30 indivíduos visitando flores de Crataeva benthami Eichl. e Kigelia africana Benth., respectivamente. Nesses dois casos, o número de flores abertas, fornecendo alimento em abundância, pode ser um fator que justifique o elevado número de morcegos em um mesmo vegetal. Provavelmente, com a redução do número de flores abertas e, conseqüentemente, uma diminuição da oferta de alimento, o número de indivíduos tenderá a se reduzir, passando as visitas a serem solitárias ou em dupla.

Para Phyllostomus hastatus o padrão de visitas em grupo é o esperado (Carvalho 1960; Goodwin \& Greenhall 1961), porém, neste estudo observou-se que esta espécie também pode visitar flores em dupla.

\section{REFERÊNCIAS BIBLIOGRÁFICAS}

BAKER, H.G. 1973. Evolutionary relationships between plants and animals in American and African forest, p. 145-159. In: B.J. MEgGers; E.S. AYENSU \& W.D. Duckworth (Eds). Tropical forest ecosystems in Africa and South American: A Comparative review. Washington, Smithsoniam Institution Press, 350p.

Carvalho, C.T. 1960. Das visitas de morcegos as flores (Mammalia, Chiroptera). An. Acad. Bras. Ci. 32 (3/4): 359-377.

1961. Sobre os hábitos alimentares de Phyllostomideos (MammaliaChiroptera). Rev. Biol. Trop. 9 (1): 53-60.

Dobat, K. \& T. Piekert-Holle. 1985. Blüten und Fledermäuse Bestäubung durch Fledermäuse und Flughunde (Chiropterophilie). Waldemar Kramer Verlag, 370p.

FAegri, K. \& L. VAN DER PIJL. 1971. The principles of pollination ecology. 
New York, Pergamon Press, 291 p.

Fischer, E.A.; F.A. Jimenez \& M. Sazima. 1992. Polinização por morcegos em duas espécies de Bombacaceae na Estação Ecológica da Juréia, São Paulo. Revta bras. Bot. 15 (1): 67-72.

Gardner, A.L. 1977. Feeding habits, p.293-350. In: R.J. BAKER; J.K. Jones JR. \& D.C. CARTER (Eds). Biology of bats of the new world family Phyllostomatidae. Special Publ. Mus. Texas tech Univ. 13: 1-364.

GuimarÃES, J.L. 1951. Aspectos geobotânicos e ecológicos do Km 47 da rodovia Rio-São Paulo. Arq. Serv. Florestal., Rio de Janeiro, 5: 35-70.

Goodwin, G.G. \& A.M. Greenhall. 1961. A review of bats of Trinidad and Tobago. Bull. Amer. Mus. Nat. Hist. 122 (3): 187-301.

Heithaus, E.R. 1982. Coevolution between bats and plants, p. 327-367. In: T.H. Kunz (Ed.). Ecology of bats. New York, Plenum Press, 425p.

Howell, D.J. 1974. Acoustic behaviour and feeding in Glossophaginae bats. J. Mammal. 55 (2): 293-308

Howell, D.J. \& D. Burch. 1974. Food habits of some Costa Rican bats. Rev. Biol. Trop. 21 (2): 281-294.

Howell, D.J. \& N. Hodking. 1976. Feeding adaptation in the hair and tongues of néctar-feeding bats. Jour. Mammal. 148: 329-336.

LEMKE, T.O. 1984. Foraging ecology of the long-nosed bat Glossophaga soricina, with respect to resource availability. Ecology 65 (2): 538-548.

Ramirez, N.; C. Sobrevila; N.X. Enrech \& T. Ruiz-Zapata. 1984. Floral biology and breeding systems of Bauhinia ungulata Tamb (Leguminosae), a bat pollinated tree in Venezuela Lhanos. Amer. Jour. Bot. 71 (2): 273-280.

SAZIMA, 1. \& M. SAZIMA. 1977. Solitary and group foraging: Two flower-visit patterns of the lesser spear-nosed bat Phyllostomus discolor. Biotropica 9 (3): 213-215.

SAZIMA, M. \& I. SAZIMA. 1980. Bats visit to Macgravia myriostigma Tr. et Planch (Marcgraviaceae) in Southeastern Brazil. Flora 169: 84-88.

Sazima, M.; M.E. Fabian \& I. Sazima. 1982. Polinização de Luehea speciosa (Tiliaceae) por Glossophaga soricina (Chiroptera- Phyllostomidae). Rev. Bras. Biol. 42 (3): 505-513.

SILVA, S.S.P. 1991. Utilização de recursos florais na alimentação de morcegos filostomídeos no Campus da Universidade Federal Rural do Rio de Janeiro, Itaguaí - RJ. Tese Mestrado, não publicada, Universidade Federal do Paraná, Curitiba, 88p. 